Progress in Nuclear Science and Technology

Volume 6 (2019) pp. 117-121

\title{
ARTICLE
}

\section{AMPX MG library of JENDL-4.0}

\author{
Chikara Konno* \\ Japan Atomic Energy Agency, 2-4 Shirakata, Tokai-mura, Naka-gun, Ibaraki-ken, 319-1195, Japan
}

\begin{abstract}
SCALE6.2.1 was released in 2016 and has been used worldwide. It includes new AMPX multigroup format files (AMPX MG libraries) of ENDF/B-VII.0 and ENDF/B-VII.1 and a new nuclear data processing code AMPX-6, which produces AMPX MG libraries. Thus we have produced an AMPX MG library (neutron 200 groups and gamma 47 groups) of JENDL-4.0 in order to disseminate JENDL-4.0. Neutron and gamma spectra inside a sphere of $1 \mathrm{~m}$ in radius with a $20 \mathrm{MeV}$ neutron source at the center were calculated with ANISN and the iron experiment at JAEA/FNS was analyzed with DORT, in order to validate the AMPX MG library of JENDL-4.0. As a result, it was verified that the AMPX MG library of JENDL-4.0 had no problems except for the self-shielding correction which is a common problem in AMPX MG libraries.
\end{abstract}

Keywords: SCALE6.2.1; AMPX MG library; JENDL-4.0; AMPX-6; ExSite; self-shielding correction

\section{Introduction}

There are two major multigroup (MG) format files of nuclear data libraries. One is a MATXS file [1], the other is an AMPX MG file [2]. The MATXS file can be produced with the NJOY [1] code and several MATXS files of the JENDL [3], ENDF/B [4] and JEFF [5] nuclear data libraries have been released [6]. On the other hand, it is not so easy to produce AMPX MG files (AMPX MG libraries) because a processing tool for AMPX MG files is not completely released so far. In 2016, the SCALE6.2.1 code system [2] was released and has been used worldwide. It includes new AMPX MG libraries of ENDF/B-VII.0 [7] and ENDF/B-VII.1 [4] and a new nuclear data processing code AMPX-6 [8], which produces AMPX MG libraries.

At ISORD5 we pointed out that the self-shielding correction in VITAMIN-B6 [9] (AMPX MG library of ENDF/B-VI.3 [10]) was not adequate [11] as briefly described in Sec. 2. One of reasons of this problem is related to a weighting function adopted in AMPX MG files. We are interested in whether current AMPX MG libraries resolve the problem on the self-shielding correction.

Thus we produce an AMPX MG library of JENDL-4.0 [3] (AMPX MG J40) in order to disseminate JENDL-4.0 and examine whether AMPX MG files can correct the self-shielding effect adequately or not by using AMPX MG J40.

*Corresponding author. Email: konno.chikara@jaea.go.jp

\section{Overview of VITAMIN-B6 problems}

VITAMIN-B6 is an AMPX MG library of ENDF/B-VI release 3, the group structure of which is 199 groups for neutron and 42 groups for gamma. The problems in VITAMIN-B6 have been reported in Ref. 11 in details. Here they are explained briefly by using a simple calculation.

The calculation model is shown in Figure 1; a natural iron sphere of $1 \mathrm{~m}$ in radius with an isotropic neutron source of 17.332-19.64 $\mathrm{MeV}$ (the first group in VITAMIN-B6). Neutron spectra in the sphere were calculated with a one-dimensional Sn code ANISN [12] and a multigroup library produced from VITAMIN-B6 by using the SCAMPI [13] code. Two more calculations were carried out for comparison. One was another ANISN calculation with a multigroup library produced from FENDL/MG-1.1 [14], which was a MATXS file of FENDL-1.1 [15] and iron data of which were taken from ENDF/B-VI, by using the TRANSX2 [16] code. The other was a reference calculation with the Monte Carlo code MCNP4C [17] and the official ACE file [17] of ENDF/B-VI. Figure 2 shows the calculated neutron

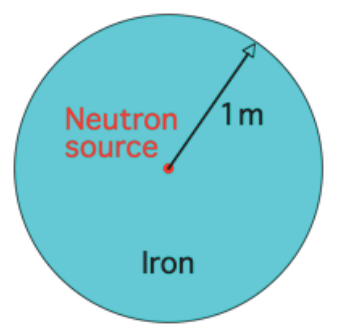

Figure 1. Calculation model. 


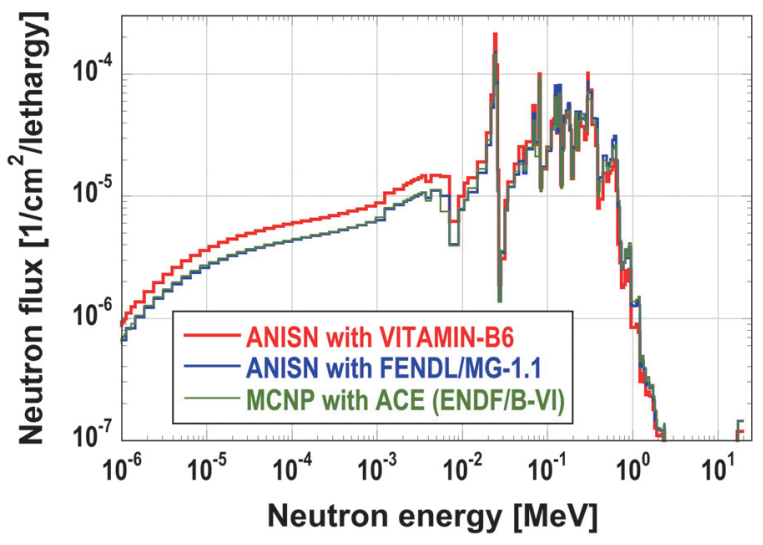

Figure 2. Neutron spectra at $60 \mathrm{~cm}$ from iron sphere center.

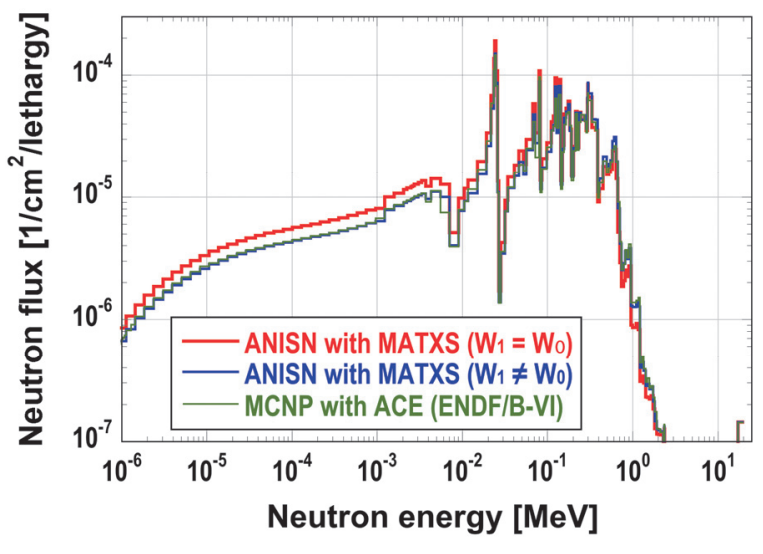

Figure 3. Neutron spectra at $60 \mathrm{~cm}$ from iron sphere center (effect of weighting flux).

spectra at the distance of $60 \mathrm{~cm}$ from the center of the iron sphere. The neutron spectrum with ANISN and VITAMIN-B6 is by at most $50 \%$ larger than that with MCNP, while that with ANISN and FENDL/MG-1.1 agrees with that with MCNP.

Our detailed investigation on VITAMIN-B6 specified that the following two problems caused the above discrepancy in the neutron flux.

1) The smallest background cross section of 1 barn is larger than that $(0.2$ barn $)$ required for natural iron.

2) The weighting function $W_{\ell}(E)$ of $C(E) /\left(\sigma_{t}+\sigma_{0}\right)$, where $\mathrm{C}(\mathrm{E})$ is a smooth part, $\sigma_{\mathrm{t}}$ is the total cross section and $\sigma_{0}$ is the background cross section, is adopted, not the weighting function of $\mathrm{C}(\mathrm{E}) /\left(\sigma_{\mathrm{t}}+\sigma_{0}\right)^{\ell+1}$, where $\ell$ is a Legendre expansion order.

The problem 1) is only for VITAMIN-B6, but all AMPX MG files have the problem 2) because they are produced by using the weighting function of $\mathrm{C}(\mathrm{E}) /\left(\sigma_{\mathrm{t}}+\sigma_{0}\right)$. Note that MATXS files do not have the problem 2) because they are produced by using the weighting function up to $\mathrm{C}(\mathrm{E}) /\left(\sigma_{\mathrm{t}}+\sigma_{0}\right)^{2}$. The same calculation as Figure 2 was carried out by using multigroup libraries of $\mathrm{W}_{1}(\mathrm{E})=\mathrm{W}_{0}(\mathrm{E})$ [abnormal usage in TRANSX2] and

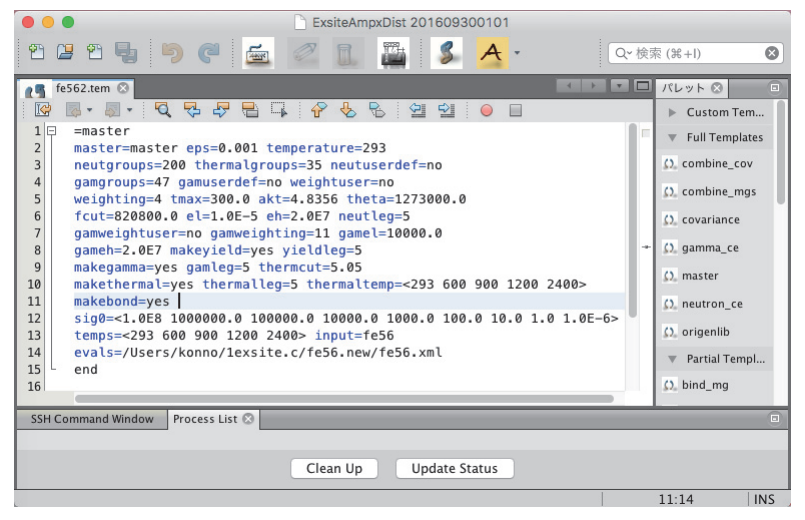

Figure 4. ExSite GUI window.

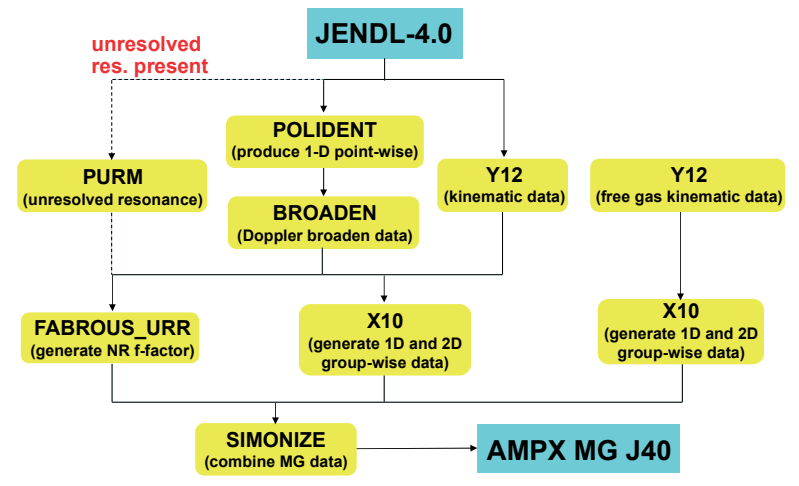

Figure 5. AMPX-6 processing flow for neutron only.

$\mathrm{W}_{1}(\mathrm{E}) \neq \mathrm{W}_{0}(\mathrm{E})$ [normal usage in TRANSX2] produced from the MATXS file in Figure 2 in order to specify the effect of the problem 2). Figure 3 shows the effect of the weighting function in the neutron spectra at $60 \mathrm{~cm}$ from the iron sphere center. The neutron spectrum with the MATXS file of $\mathrm{W}_{1}(\mathrm{E})=\mathrm{W}_{0}(\mathrm{E})$ is different from that with the MATXS file of $\mathrm{W}_{1}(\mathrm{E}) \neq \mathrm{W}_{0}(\mathrm{E})$, which agrees with that with MCNP. It is noted that the effect of the problem 2) is rather large.

\section{How to produce AMPX MG J40}

The AMPX-6 code in SCALE6.2.1 was used to produce AMPX MG J40. The specifications of AMPX MG J40 was almost the same as those of V7.1-200N47G [2] in SCALE6.2.1, which are as the followings,

1) group structure : neutron 200 groups, gamma 47 groups,

2) smooth part of weighting function : a Maxwellian $1 / E$ - fission - 1/E spectrum for neutron and a $1 / E$ spectrum for gamma except for actinides, where the neutron part is a PWR spectrum,

3) Legendre polynomial expansions of scattering cross sections : $\mathrm{P}_{5}$ order,

4) background cross section [barn] : Nuclei $\leq{ }^{90} \mathrm{Y}: 10^{8}, 10^{6}, 10^{5}, 10^{4}, 10^{3}, 10^{2}, 10,1,10^{-6}$, 


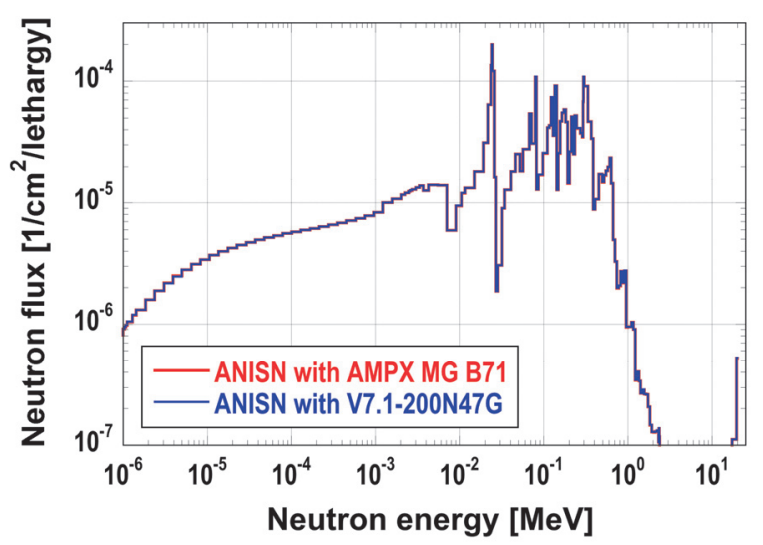

(a) Neutron spectra at $60 \mathrm{~cm}$ from iron sphere center

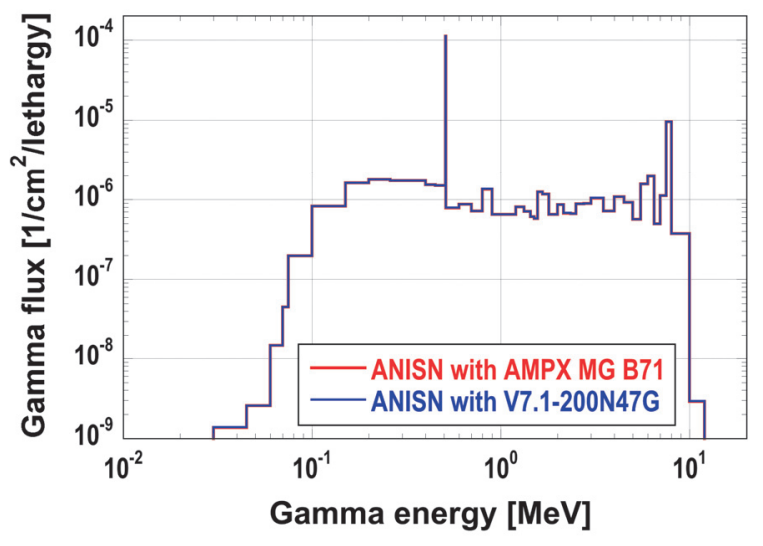

(b) Gamma spectra at $60 \mathrm{~cm}$ from iron sphere center

Figure 6. Comparison of calculation results with AMPX MG B71 and V7.1-200N47G for iron sphere.

Nuclei $\geq{ }^{90} \mathrm{Zr}: 10^{8}, 10^{6}, 10^{5}, 2 \times 10^{4}, 10^{4}, 5 \times 10^{3}$, $2 \times 10^{3}, 10^{3}, 640,320,160,120,8060,40,30,2915$, $10,8,6,4,2,1,0.01,10^{-6}$,

5) temperature $[\mathrm{K}]: 293,600,900,1200,2400$.

Because the PWR spectrum for actinides in the smooth part of the weighting function in V7.1-200N47G was not opened, we also used the smooth part of the weighting function for actinides in AMPX MG J40. The problem 1) in VITAMIN-B6 does not occur because the lowest background cross section is $10^{-6}$ barn.

Input data of AMPX-6 were generated with an auxiliary code ExSite [8] in AMPX-6. ExSite has a GUI interface as shown in Figure 4 and ExSite generates full input data of AMPX-6 from basic data as shown in Figure 4. The processing flow of AMPX-6 is shown in Figure 5. The main processing functions of AMPX-6 are similar to those of NJOY. All the nuclei of JENDL-4.0 were automatically processed by using a UNIX shell-script. In order to verify the input data and procedure, several nuclei in ENDF/B-VII.1 were also produced (AMPX MG B71).

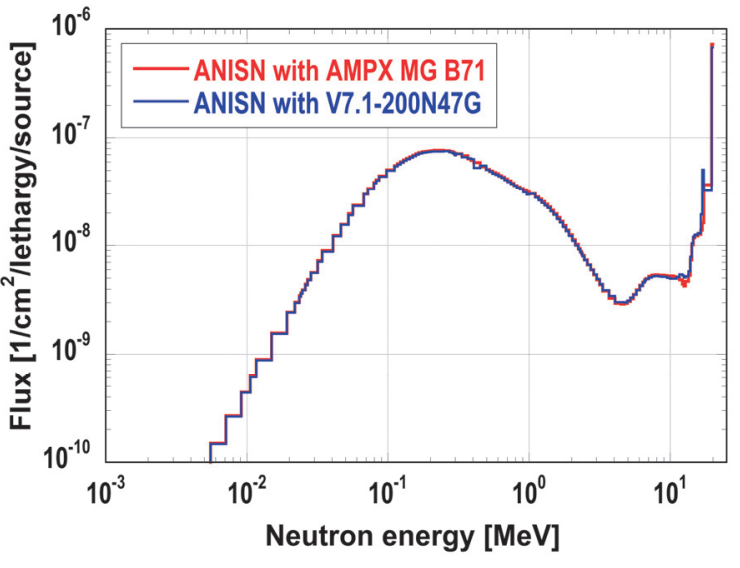

(a) Neutron spectra at $60 \mathrm{~cm}$ from protactinium sphere center

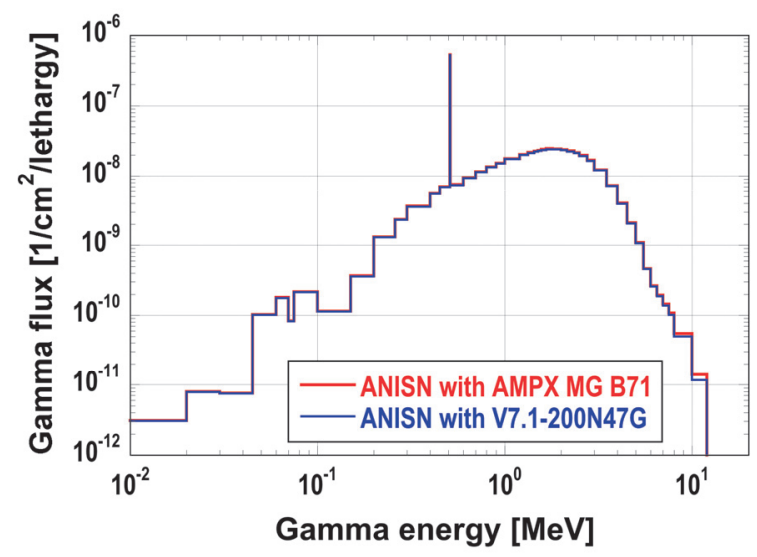

(b) Gamma spectra at $60 \mathrm{~cm}$ from protactinium sphere center

Figure 7. Comparison of calculation results with AMPX MG B71 and V7.1-200N47G for protactinium sphere.

\section{Verification of input data and procedure}

Neutron and gamma spectra inside a sphere of $1 \mathrm{~m}$ in radius with an isotropic neutron source of $19.64-20$ $\mathrm{MeV}$ (the first group of neutron 200 group) at the center as shown in Figure 1 were calculated with ANISN and multigroup libraries produced from V7.1-200N47G and AMPX MG B71 by using SCALE6.2.1 to verify the input data and procedure of AMPX-6 for AMPX MG J40. Figure 6 shows the result of an iron sphere, where the self-shielding effect is large. In Figure 6 the calculated neutron and gamma spectra with AMPX MG B71 agree with those with V7.1-200N47G very well.

Figure 7 shows the result of a protactinium sphere, where the smooth part of the weighting function is different between V7.1-200N47G and AMPX MG B71. The calculated neutron and gamma spectra with AMPX MG B71 are slightly different from those with V7.1-200N47G. It is considered that the small difference comes from the different smooth part of the weighting function.

It is verified that the input data and procedure of AMPX-6 for AMPX MG J40 have no problems. 


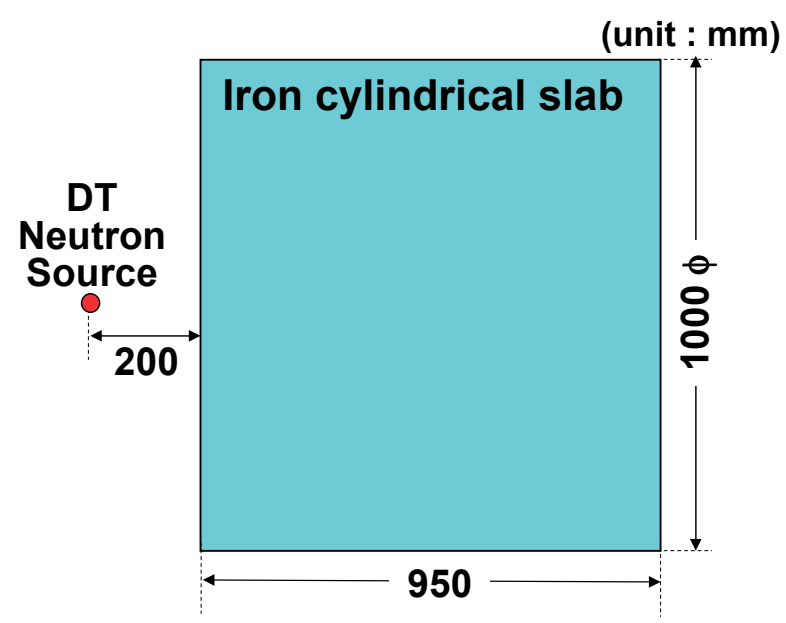

Figure 8. Experimental configuration of iron experiment at JAEA/FNS.

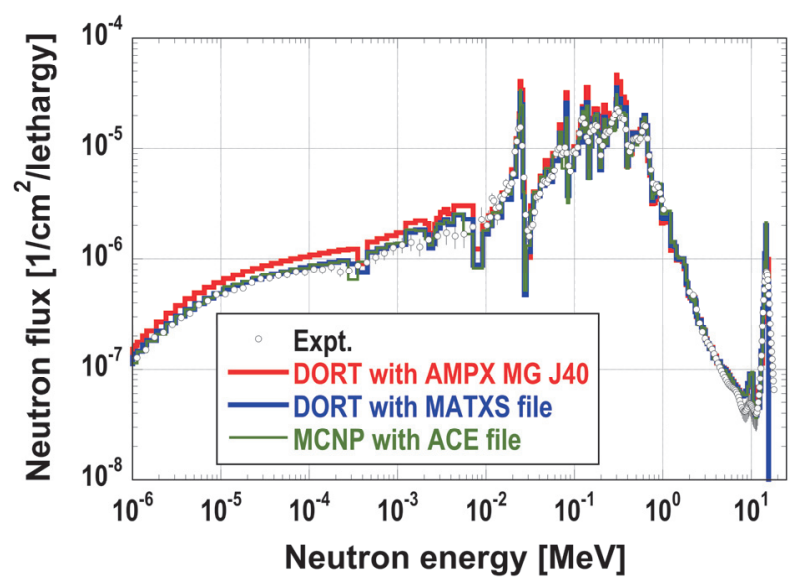

Figure 9. Neutron spectra at depth of $410 \mathrm{~mm}$ inside iron slab.

\section{Self-shielding correction test}

In order to examine the self-shielding correction in AMPX MG libraries, the iron shielding experiment [18] at the Fusion Neutronics Source (FNS) facility in Japan Atomic Energy Agency (JAEA) was analyzed with a two-dimensional Sn code DORT [12] and AMPX MG J40. Figure 8 shows the experimental configuration of the iron experiment. Neutron spectra and reaction rates of dosimetry reactions were measured inside the iron slab. This experiment was analyzed with a two-dimensional Sn code DORT [12] and AMP MG J40. A MATXS file of JENDL-4.0 produced with NJOY was also used for comparison. Moreover a calculation with MCNP4C and the official JENDL-4.0 ACE file (AceLibJ40) [19] was carried out as a reference because the self-shielding correction was automatically done in MCNP calculations. Figure 9 shows the measured and calculated neutron spectra at the depth of $410 \mathrm{~mm}$ inside the iron slab. The neutron spectra calculated with DORT and AMPX MG J40 is different from the measured one and calculated ones with DORT and the MATXS file, and MCNP and AceLibJ40. It is concluded that the self-shielding correction in AMPX MG libraries is still inadequate.

\section{Conclusion}

An AMPX MG library of JENDL-4.0 was produced with AMPX-6 in order to disseminate JENDL-4.0. The specifications of the AMPX MG library were the same as those of V7.1-200N47G, except for the smooth part of the weighting function. From test calculations with a simple model and an analysis of the iron experiment at JAEA/FNS, it was confirmed that the AMPX MG library of JENDL-4.0 had no problem except for the self-shielding correction. It is noted that the insufficient self-shielding problem in AMPX MG libraries, which was pointed out before, still remains. AMPX MG J40 will be released through JAEA website for SCALE users.

\section{Acknowledgements}

The author wishes to thank Dr. D. Wiarda, ORNL, for useful information on V7.1-200N47G and AMPX-6.

\section{References}

[1] A.C. Kahler (Ed.), The NJOY Nuclear Dara Processing System, Version 2016, LA-UR-17-20093, Los Alamos National Laboratory, (2016).

[2] B.T. Rearden and M.A. Jessee (Ed.), SCALE Code System, ORNL/TM-2005/39 version 6.2.1, Oak Ridge National Laboratory, (2016).

[3] K. Shibata, O. Iwamoto, T. Nakagawa, N. Iwamoto, A. Ichihara, S. Kunieda, S. Chiba, K. Furutaka, N. Otuka, T. Ohasawa, T. Murata, H. Matsunobu, A. Zukeran, S. Kamada and J. Katakura, JENDL-4.0: A new library for nuclear science and engineering, $J$. Nucl. Sci. Technol. 48 (2011), pp. 1-30.

[4] M.B. Chadwick, M. Herman, P. Oblozinsky, M.E. Dunn, Y. Danon, A.C. Kahler, D.L. Smith, B. Pritychenko, G. Arbanas, R. Arcilla, R. Brewer, D.A. Brown, R. Capote, A.D. Carlson, Y.S. Cho, H. Derrien, K. Guber, G.M. Hale, S. Hoblit, S. Holloway, T.D. Johnson, T. Kawano, B.C. Kiedrowski, H. Kim, S. Kunieda, N.M. Larson, L. Leal, J.P. Lestone, R.C. Little, E.A. McCutchan, R.E. MacFarlane, M. MacInnes, C.M. Mattoon, R.D. McKnight, S.F. Mughabghab, G.P.A. Nobre, G. Palmiotti, A. Palumbo, M.T. Pigni, V.G. Pronyaev, R.O. Sayer, A.A. Sonzogni, N.C. Summers, P. Talou, I.J. Thompson, A. Trkov, R.L. Vogt, S.C. van der Marck, A. Wallner, M.C. White, D. Wiarda and P.G. Young, ENDF/B-VII .1 Nuclear data for science and technology: cross sections, covariances, fission product yields and decay data, Nucl. Data Sheets 112 (2011), pp. 2887-2996. 
[5] A. Koning, R. Forrest, M. Kellett, R. Mills, H Henriksson and Y. Rugama (Ed.), The JEFF-3.1 Nuclear Data Library, JEFF Report 21, OECD Nuclear Energy Agency, (2006).

[6] http://atom.kaeri.re.kr/NDVG/.

[7] M.B. Chadwick, P. Oblozinsky, M. Herman, N.M. Greene, R.D. McKnight, D.L. Smith, P.G. Young, R.E. MacFarlane, G.M. Hale, S.C. Frankle, A.C. Kahler, T. Kawano, R.C. Little, D.G. Madland, P. Moller, R.D. Mosteller, P.R. Page, P. Talou, H. Trellue, M.C. White, W.B. Wilson, R. Arcilla, C.L. Dunford, S.F. Mughabghab, B. Pritychenko, D. Rochman, A.A. Sonzogni, C.R. Lubitz, T.H. Trumbull, J.P. Weinman, D.A. Brown, D.E. Cullen, D.P. Heinrichs, D.P. McNabb, H. Derrien, M.E. Dunn, N.M. Larson, L.C. Leal, A.D. Carlson, R.C. Block, J.B. Briggs, E.T. Cheng, H.C. Huria, M.L. Zerkle, K.S. Kozier, A. Courcelle, V. Pronyaev and S.C. van der Marck, ENDF/B-VII.0 : next generation evaluated nuclear data library for nuclear science and technology, Nucl. Data Sheets 107 (2006), pp. 2931-3059.

[8] D. Wiarda, M.E. Dunn, N.M. Greene, M.L. Williams, C. Celik and L.M. Petrie, $A M P X-6 A$ Modular Code System for Processing ENDF/B, ORNL/TM-2016/43, Oak Ridge National Laboratory, (2016).

[9] J.E. White, D.T. Ingersoll, J.E. White, R.Q. Wright, H.T. Hunter, C.O. Slater, N.M. Greene, R.E. MacFarlane and R.W. Roussin, Production and Testing of the VITAMIN-B6 Fine-Group and the BUGLE-93 Broad-Group Neutron/Photon Cross-Section Libraries Derived from ENDF/B-VI Nuclear Data, ORNL-6795, NUREG/CR-6214, Oak Ridge National Laboratory, (1995).

[10]P.F. Rose, ENDF/B-VI Summary Documentation, BNL-NCS-17541 (ENDF-201), Brookhaven National Laboratory, (1991).

[11]C. Konno, K. Ochiai and S. Ohnishi, Insufficient self-shielding correction in VITAMIN-B6, Prog. in
Nucl. Sci. Technol. 1 (2011), pp. 32-35.

[12]DOORS3.2a: One, Two- and Three-Dimensional Discrete Ordinates Neutron/Photon Transport Code System, RSICC CODE PACKAGE CCC-650 (2007).

[13] S.M. Bowman, OFFSCALE: A PC Input Processor for the SCALE Code System Volume 1: The CSASIN Processor for the SCAMPI Code System, NUREG/CR-6182, Vol. 1 (ORNL/TM-12663/V1), Oak Ridge National Laboratory, (1995).

[14]R.E. MacFarlane, FENDL/MG, Library of Multigroup Cross-Sections in GENDF and MATXS Format for Neutron-Photon Transport Calculations, Version 1.1 of March 1995, Summary documentation by A.B. Pashchenko, H. Wienke, S. Ganesan, IAEA-NDS-129 Rev. 3, International Atomic Energy Agency, (1996).

[15]A.B. Pashchenko, H. Wienke, S. Ganesan and P.K. McLaughlin, FENDL/E, Evaluated Nuclear Data Library of Neutron Interaction Cross Sections, Photon Production Cross Sections and Photon-Atom Interaction Cross Sections for Fusion Applications, Version 1.1 of November 1994, IAEA(NDS)-128, Rev. 3, International Atomic Energy Agency, (1996).

[16]R.E. MacFarlane, TRANSX 2: A Code for Interfacing MATXS Cross-Section Libraries to Nuclear Transport Codes, LA-12312-MS, Los Alamos National Laboratory, (1993).

[17]J.F. Briesmeister (Ed.), MCNP - A General Monte Carlo $N$-Particle Transport Code, Version 4C, LA-13709-M, Los Alamos National Laboratory, (2000).

[18] F. Maekawa, C. Konno, Y. Kasugai, Y. Oyama and Y. Ikeda, Data Collection of Fusion Neutronics Benchmark Experiment Conducted at FNS/JAEA, JAERI-Data/Code 98-021, Japan Atomic Energy Research Institute, (1998).

[19]http://prodas.jaea.go.jp/?AceLibJ40 [in Japanese]. 